\title{
Issues in the interpretation of postmortem toxicology
}

\author{
Roger W. Byard · Danielle M. Butzbach
}

Published online: 15 September 2011

(C) Springer Science+Business Media, LLC 2011

From the earliest times humans have utilized a wide variety of manufactured and natural substances to treat disease [1], to facilitate religious ritual and to augment recreational activities. In modern Western societies the range of prescription, non-prescription and illicit drugs that are available is probably greater than in any previous time and so the challenge for toxicology laboratories to identify specific substances is considerable. Cases that involve drugs that are encountered in forensic practice are varied, ranging from inadvertent deaths due to the direct toxic actions of a drug, to exacerbations of underlying disease, and to suicides and homicides.

The role of postmortem toxicology is significant as it can provide vital information on possible causes of death related to illicit or prescription drugs or poisons. It can also be a useful way of determining the types of illicit drugs that are being used in a particular community, and to provide information to law enforcement agencies, physicians and users when new drugs with significant side effects such as paramethoxyamphetamine are being introduced, or when purer grades of opiates such as heroin are being sold. Both may be associated with increasing cases of lethal and nonlethal drug toxicity $[2,3]$.

Determining the significance of postmortem drug levels is not however, always a straightforward exercise. Drug levels may be altered by attempted resuscitation and

\footnotetext{
R. W. Byard $(\bowtie)$

Discipline of Anatomy \& Pathology, Level 3 Medical School North Building, The University of Adelaide, Frome Road,

Adelaide 5005, Australia

e-mail: roger.byard@sa.gov.au

D. M. Butzbach

School of Chemical and Physical Sciences, Flinders University, Bedford Park, Adelaide, SA, Australia
}

changes may occur in drug levels after death that are not predictable. Variations in sampling may also significantly affect measurements of levels of target medications and drugs [4]. Postmortem redistribution of drugs is a well recognized phenomenon with blood levels increasing if drugs equilibrate between adjacent tissues and blood. For example, changes in tissue levels have been shown experimentally to occur due to passive diffusion from the adjacent stomach [5]. For this reason it is standard practice at autopsy to sample peripheral blood, with the femoral vein being the most appropriate site. Tissue levels may be affected by postmortem interval, refrigeration before autopsy and the position of the body [6]. Levels of amitriptyline may increase by a factor of 3.9, and methadone by 2.6 in postmortem blood [4]. Analysis of ante-mortem specimens from, for example, hospital emergency departments, will provide measurements that have not been affected by postmortem processes.

Another issue concerns the range of so-called "routine" drug screening in forensic toxicology laboratories. Not all drugs or toxins can be screened or tested for, and so a negative result does not necessarily exclude a particular substance. This is particularly a concern where targeted screening is relied upon, as these methods would likely fail to detect unknown compounds such as new designer drugs. On occasion levels may be extremely difficult to measure and so quantification will not be possible [7].

It must be remembered that a corpse is not a static environment. Tissue and organ metabolism continues after terminal cardiorespiratory arrest which may significantly alter drug levels. For example, gamma hydroxybutyrate (GHB) may be formed after death from the endogenous metabolism of the neurotransmitter gamma-aminobutyric acid, increasing blood levels by up to $100 \mathrm{mg} / \mathrm{L}$ [4]. In addition the process of decomposition involving both 
autolysis and putrefaction may increase or reduce levels. The postmortem generation of alcohol by bacteria is well recognised, but also bacteria may break down drugs and their metabolites with progressive diminution in levels until detection may no longer be possible [8]. Storage may decrease drug or poison levels, an example of which is malathion, an insecticide that is broken down by the preservative sodium fluoride [9]. Other substances such as volatile inhalants may disperse from the body very rapidly after death. Increases in drug concentrations for tissues and organs may also occur during decomposition due to release of fluids and subsequent decrease in tissue mass [10].

Once toxicology results are available a further range of complex issues arises. Determining what constitutes a lethal level may be extremely difficult in some drugs due to great overlap between non-toxic, therapeutic and lethal levels. There may also be habituation to the effects of a drug so that high levels can be tolerated. Good examples are some of the ring-derivative amphetamines and opiates [11, 12]. Users may also have ingested a number of drugs that may have synergistic effects. Whether levels of drugs measured in the terminally ill are a true indication of levels that were present prior to possible agonal dehydration with hemoconcentration may also not be determinable.

Drugs may also have potentially lethal effects if they exacerbate underlying organic conditions, and so full evaluation of cases at autopsy requires the careful documentation of natural diseases and the determination of whether drugs were involved with terminal mechanisms. For example, diabetic ketoacidosis may be provoked by drug overdose, and upper airway narrowing may be increased by the relaxant effects of narcotics $[13,14]$.

Pediatric cases present particular problems. Despite the recognized possibility of alterations in drug levels in cardiac blood after death from redistribution, blood is usually taken from the hearts in infants for toxicology as there is so little peripheral blood available. Given the possibility of postmortem redistribution, the validity of these measurements could be questioned. Another issue that may arise in infancy is in determining the precise significance of a particular drug level, as standard therapeutic ranges are generally based on adult studies. Whether these standards apply to the very young may be unclear. For example, could a therapeutic level of an antihistamine that would have no effect on an adult be a significant compounding factor in an infant predisposed to sudden infant death syndrome? In the United States the National Association of Medical Examiners (NAME) is compiling a registry of pediatric drug levels to assist with these issues.

Organic toxins are technically difficult for laboratories to detect, even when the particular substance is known [15]. An additional problem concerns herbal medicines which may be contaminated by heavy metals or pesticides that may not be routinely tested for, may have more toxic but cheaper ingredients substituted, or may cause lethal illness in their own right. Another issue concerns the potential interaction of certain herbs with prescription medications, examples of which include St John's Wort, which may interfere with the effects of warfarin, leading to thrombosis. The extent of this problem is unfortunately not known at present as screening for herbal substances is not routinely performed in most laboratories [16, 17].

Every so often a case arises where death was assumed to be due to obvious underlying disease (often cardiovascular), only to find that toxicology has revealed a lethal level of a particular drug. Such cases raise the question as to what extent toxicology should be undertaken. Should it be on every case and if so how extensive should it be? These questions are generally unresolvable; however the interpretation of postmortem toxicology requires a clear understanding of its limitations. In cases where quite complex drug interactions may have occurred the early involvement of a clinical toxicologist may be the most useful step for a pathologist to take.

\section{References}

1. Byard RW. Traditional medicine of aboriginal Australia. Can Med Assoc J. 1988;139:792-4.

2. Byard RW, Gilbert J, James R, Lokan RJ. Amphetamine derivative fatalities in South Australia-is "Ecstasy" the culprit? Am J Forensic Med Pathol. 1998;19:261-5.

3. Caldicott DGE, Edwards NA, Kruys A, Kirkbride KP, Sims DN, Byard RW, Prior M, Irvine R. Dancing with 'death'; P-methoxyamphetamine overdose and its acute management. J Tox Clin Tox. 2003;41:143-54.

4. Skopp G. Postmortem toxicology. Forensic Sci Med Pathol. 2010;6:314-25.

5. Pounder DJ, Fuke C, Cox DE, Smith D, Kuroda N. Postmortem diffusion of drugs from gastric residue: an experimental study. Am J Forensic Med Pathol. 1996;17:1-7.

6. Pounder DJ, Adams E, Fuke C, Langford AM. Site to site variability of post-mortem drug concentration in liver and lung. J Forensic Sci. 1996;41:927-32.

7. Kostakis C, Byard RW. Sudden death associated with intravenous injection of toad extract. Forensic Sci Int. 2009;188:e1-5.

8. Butzbach D, Byard RW, Kobus H, Lokan R, Sims DN, Stockham P, Walker GS. Stability of selected psychiatric drugs during decomposition. Paper presented at $20^{\text {th }}$ International Symposium on the Forensic Sciences (ANZFSS), Sydney, Australia. September 2010.

9. Pannell M, Gilbert JD, Gardiner J, Byard RW. Death due to malathion poisoning. J Clin Forensic Med. 2001;8:156-9.

10. Wyman JF, Dean DE, Yinger R, Simmons A, Brobst D, Bissell M, Silveira R, Kelly N, Shott R, Ohr J, Howard R, Lewis B. The temporal fate of drugs in decomposing porcine tissue. J Forensic Sci. 2011;56:694-9.

11. Milroy CM. "Ecstasy" associated deaths: what is a fatal concentration? Analysis of a case series. Forensic Sci Med Pathol. 2011;7:248-52. 
12. Green H, James RA, Gilbert J, Harpas P, Byard RW. Methadone maintenance programs - a two edged sword? Am J Forensic Med Pathol. 2000;21:359-61.

13. Byard RW, Riches KJ, Kostakis C, Felgate HE. Diabetic ketoacidosis-A possible complicating factor in deaths associated with drug overdose: two case reports. Med Sci Law. 2006;46:81-4.

14. Byard RW, Gilbert JD. Narcotic administration and stenosing lesions of the upper airway-a potentially lethal combination. J Clin Forensic Med. 2005;12:29-31.
15. Byard RW, James RA, Felgate P. Detecting organic toxins in possible fatal poisonings-a diagnostic problem. J Clin Forensic Med. 2002;9:85-8.

16. Byard RW. The potential forensic significance of traditional herbal medicines. J Forensic Sci. 2010;55:89-92.

17. Byard RW, Musgrave I. Herbal medicines and forensic investigations. Forensic Sci Med Pathol. 2010;6:81-2. 